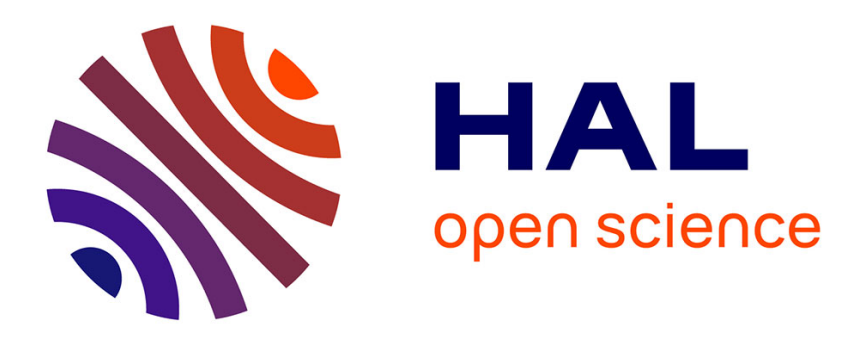

\title{
Colloque international de magnétisme. Avant-propos L. Néel
}

\section{To cite this version:}

L. Néel. Colloque international de magnétisme. Avant-propos. J. Phys. Radium, 1959, 20 (2-3), pp.65-69. 10.1051/jphysrad:01959002002-306500 . jpa-00236070

\section{HAL Id: jpa-00236070 https://hal.science/jpa-00236070}

Submitted on 1 Jan 1959

HAL is a multi-disciplinary open access archive for the deposit and dissemination of scientific research documents, whether they are published or not. The documents may come from teaching and research institutions in France or abroad, or from public or private research centers.
L'archive ouverte pluridisciplinaire HAL, est destinée au dépôt et à la diffusion de documents scientifiques de niveau recherche, publiés ou non, émanant des établissements d'enseignement et de recherche français ou étrangers, des laboratoires publics ou privés. 


\section{LE JOURNAL DE PHYSIQUE}

\section{LE RADIUN}

\section{GOLLOQUE INTERNATIONAL DE MAGNÉTISME}

\section{AVANT-PROPOS}

Le Colloque International de Magnétisme dont les comptes rendus sont présentés plus loin s'est tenu à Grenoble du 2 au 6 juillet 1958. C'est la troisième réunion internationale importante consacrée en France au Magnétisme. La première, organisée par P. Weiss à Strasbourg du 21 au 25 mai 1939 et où une vingtaine de communications furent discutées, connut un très beau succès mais la diffusion des résultats fut presque complètement arrêtée par la guerre. La seconde fut le Colloque International sur le Ferromagnétisme et l'Antiferromagnétisme organisé à Grenoble du 3 au 6 juillet $1950: 49$ communications y furent présentées. Nous devons citer encore le Colloque National de Magnétisme commémoratif de l'œuvre de Pierre Weiss organisé à Strasbourg du 8 au 10 juillet 1957 et publié par les soins du Centre National de la Recherche Scientifique.

Le Colloque de 1958 a réuni 200 auditeurs dont 150 étrangers comprenant les spécialistes les plus éminents du magnétisme : 78 rapports et communications furent discutés.

Ce Colloque a été organisé avec le concours financier de l'U. N. E. S. C. O., sous les auspices de l'Union Internationale de Physique Pure et Appliquée et de son Comité Exécutif. Nous avons reçu des subventions de $M$. le Directeur Génêral des Affaires Culturelles et Techniques, au Mınistère des Affaires Étrangères, et de M. le Président du Conseil Supérieur de la Recherche Scientifique et du Progrès Technique.

Nous avons été également aidés par l'Institut de Recherches de la Sidérurgie, parl'Association UgineAllevard pour la fabrication et l'exploitation des aimants permanents, par la Compagnie Générale de Télégraphie sans Fil, ainsi que par la Société des Forges et Ateliers du Creusot.
Enfin, la publication des rapports, des communications et des discussions a été assurée par le Journal de Physique, avec le concours du Centre National de la Recherche Scientifique.

Nous tenons à remercier chaleureusement ces institutions et organismes de l'ajde matérielle et morale qu'ils nous ont apportée et qui a facilité considérablement notre tâche. Nous ne voudrions pas non plus oublier dans nos remerciements tout le personnel du Laboratoire d'Électrostatique et de Physique du Métal dont le dévouement inlassable a permis l'organisation matérielle de cette manifestation.

Il n'est pas possible de résumer ici, même brièvement, les différentes communications qui furent discutées au cours de 30 heures de séances. Selon le désir exprimé par l'Assemblée générale de l'Union Internationale de Physique pure et appliquée, un compte rendu de quelques pages des travaux du Colloque a été publié dans le numéro de décembre 1958 de Physics Today, sous 'la signature de B. Dreyfus.

Bien que les sujets de caractère purement techniques aient été exclus, la variété des exposés fut remarquable et donna lieu à des échanges de vue passionnants. Jamais le Magnétisme n'a été plus vivant : de nouvelles voies de recherche s'ouvrent tous les jours, en liaisons toujours plus étroites avec les autres chapitres de la physique du solide. Nous souhaitons qu'une lecture même partielle de ce volume suscite de nouveaux chercheurs dans un domaine où la France s'est toujours distinguée depuis les travaux de Pierre Curie, de Paul Langevin et de Pierre Weiss.

L. NÉEL.

Membre de l'Institut, Professeur â la Faculté des Sciences de Grenoble.

le journal de Physique et le radium. - T. $20 .-$ N$^{\circ} 2-3$. février-yars 1959. 


\section{LISTE DES PARTICIPANTS AU COLLOQUE}

\section{Alle magne}

Brenner (R.), Vacuumschmelze Ab, Grüner Weg 37, Hanan.

Dammann (H.), Fabrik für Metallpulver-Werkstoffe Vogt \& Co, Passau.

Eckert (D.), Steatit Magnesia A. G., Lauf Pegnits, Bayern.

Fahlenbrach (H.), Friedrich Krupp, Widia Fabrik, Essen.

Kornetski (M.), Siemens \& Halske A. G., WWB.BF Lab., Balanstrasse 73, München.

Lutz, Technische Hochschule, München.

Perthel (R.), Institut für Magnetische Versktoffe, Iena.

Schweizerhof (S.), Leipziger Str. 4, Backnang.

Six Tus (K.), AEG Forschungsinstitut Frankfurt, Main-Hausen, Industriehof, Frankfurt.

Stablein (H.), Krupps, Widia-Fabrik, Essen.

VoIgt, Institut für Magnetische Werkstoffe, Iena.

Vogt (E.), Physikalisches Institut der Universität Marburg, Renthof 5, Marburg/Lanh.

Weddigen (C.), Ringstrasse 10, Gauting/München.

\section{Belgique}

Danner, Centre d'Étude de l'Énergie Nucléaire, 31 , rue Belliard, Bruxelles.

Hautecler, Centre d'Étude de l'Énergie Nucléaire, 31, rue Belliard, Bruxelles.

Lambeir (R.), Société Gevaert, 19, D. de Semerpont Laan, Barheiden.

Spaepen, Centre d'Étude de l'Énergie Nucléaire, 31 , rue Belliard, Bruxelles.

VAN DEN Bosch, Centre d'Étude de l'Énergie Nucléaire, 31, rue Belliard, Bruxelles.

\section{Canada}

Bloom (M.), The University of British Columbia, Vancouver 8.

\section{Etats-Unis}

Artman (J. O.), Applied Physics Laboratory, The Johns Hopkins University, Silver Spring, Maryland, U. S. A.

Bozorth (R. M.), Bell Telephone Laboratories, Murray-Hill (N. J.).
Brown (W. F.), Department of Electrical Engineering, University of Minnesota, Minneapolis 14 (Minn.).

Calmoun (B. A.), International Business Machines Corporation, Research Center, Poughkeepsie (N. Y.).

Clogston (A. M.), Bell Telephone Laboratories, Murray-Hill (N. J.).

Czerlinsky (E.), Components and Techniques Lab., Air Force Cambridge Research Center, Bedford (Mass.).

Dabbs (J.), Oak Ridge National Laboratory, Physics Division, Oak Ridge (Tenn.).

Daun t (J. G.), The Ohio State University, Department of Physics and Astronomy, Colombus 10 (Ohio).

Foner (S.), Lincoln Laboratory, Massachusetts Institute of Technology, Lexington (Mass.).

Goodenough (J.), Lincoln Laboratory, Massachusetts Institute of Technology, Lexington (Mass.).

Henry (W. E.), United States Naval Research Lab., Washington 25, D. C.

Hudson (R. P.), U. S. Department of Commerce, NBS, Washingt on 25, D.C.

JAcoBs (I. S.), General Electric Research Laboratory, P. O. Box 1088, Schenectady (N. Y.).

Kittel (C.), Department of Physics, University of California, Berkeley (Cal.).

Koenler ( $\mathrm{W}$. C.), Physics Division, Oak Ridge National Lab., Box P, Oak Ridge (Tenn.).

Levin (S. B.), U. S. Army Signal R et D Lab., Fort Monm out h (N. J.).

McGuire (T. R.), United States Naval Ordnance Lab., White Oak, Silver Spring (Maryland).

Meiklejohn (W.), General Electric Research Laboratory, Schenectady (N. Y.).

Porter (C. S.), Diamond Oidnance Fuze Lab., Washingt on 25, D. C.

Prat T, Lincoln Laboratory, Massachusetts Institute of Technology, Lexingt on (Mass.).

Sadier (E. L.), E. I. du Pont de Nemours and Company, Wilmington 98 (Delaware).

Schiömann (E.), Raytheon Manufacturing Company, Research Division, Waltham 54 (Mass.).

Shull (C. G.), Massachusetts Institute of Technology, Cambridge (Mass.).

Suhl (H.), Bell Telephone Laboratories, Murray Hill Laboratory, Murray Hill (N. J.). 
Tanenbaum (M.), Bell Telephone Laboratories, Murray-Hill (N. J.).

Tannenwald (P. E.), Lincoln Laboratory, Massachusetts Institute of Technology, Lexington (Mass.).

Tyrell, Bell Telephone Laboratories, Murray-Hil (N. J.).

Van Vleck (J. H.), The Physics Laboratories, Harvard University, Cambridge (Mass.).

\section{Grande-Bretagne}

Bacon (G. E.), Atomic Energy Research Establishment, Metallurgy Division, Harwell, Didcot, Berks.

Bates (W. L. F.), The University of Nottingham, University Park, Nottingham.

Crangle (J.), Department of Physics, The University, Sheffield 10.

Enwards (D. M.), Department of Mathematics, Queen Mary College, London.

Fuller (C. E.), Mullard Research Laboratories, Cross Oak Lane, Salfords, Nr. Redhill (Surrey).

Hahn (E. L.), The Clarendon Laboratory, Parks Road, Oxford.

Harrison (F. W.), Mullard Research Laboratories, Cross Oak lane, Salfords, Nr. Redhill (Surrey).

Hosecitz (K.), Mullard Research Laboratories, Cross Oak lane, Salfords, Nr. Redhill (Surrey).

Kunti (N.), The Clarendon Laboratory, Parks Road, Oxford.

Kosima (H.), Department of Physics, The University, Sheffield 10.

LEE (E. W.), The University of Nottingham, Department of Physics, Nottingham.

Lock, R. R. E.. Great Malvern.

Lynch (A. C.), Post Office Engineering Department, Research Station, Dollis Hill, London NW2.

Manuel (A. J.), Department of Physics, The University, Leeds 2.

Moore (A. C.), Ministry of Supply, Royal Radar Establishment, St-Andrews Road, Great Malvern.

Morrish (A. H.), The Clarendon Laboratory, Parks Road, Oxford.

Motr (N. F.), Cavendish Laboratory, Free School Lane, Cambridge.

Owen (J.), The Clarendon Laboratory, Parks Road,.Oxford.

Pearson (R. F.), Mullard Research Laboratories, Cross Oak Lane, Salfords, Nr. Redhill (Surrey).

Rhodes (P.), Department of Physics, The University, Leeds 2.

Sinclair (R. N.), University of Sheffield, Sheffield. Street (R.), University of Sheffizld, Sheffisld.

Sucksmith, University of Sheffi sld, Sheffield.

Teale (R. W.), Mullard Research Laboratories, Cross Oak Lane, Salfords, Nr. Redhill (Surrey).
Tebble (R. S.), The University, Sheffield.

Webber (R. T.), Office of Naval Research, Keysign House, 429, Oxford Street, London W1.

Williams, Research Laboratory, G. E. C. East Lane, Wembley.

Wohlfarth (E. P.), Imperial College of Science and Technology, Exhibition Road, South Kensington, London SW7.

Wolf (W. P.), The Clarendon Laboratory, Parks Road, Oxford.

\section{Israël}

Hirsch (A. A.), Department of Physics, Israël Institute of Technology, Technion, Haifa.

Shtrikman (S.), Department of Electronics, The Weismann Institute of Science, Rehovoth.

\section{Italie}

Biora (G.), Istituto Elettrotecnico Naz. "G. Ferraris "), Corso Massimo d'Azeglio 42, Torino.

Ferro (A.), Istituto Elettrotecnico Naz. «G. Ferraris, Corso Massimo d'Azeglio 42, Torino.

Gagliotti (G.), Comitato Nazionale per le Ricerche.

Montalenti (G.), Istituto Elettrotecnico Naz. "G. Ferraris ", Torino.

\section{Japon}

Hirone (T.), Tohoku University, Research Institute of Iron, Steel and other Metals, Sendai.

KaNDA (E.), The Research Institute for Iron, Steel and other Metals, Sendai.

Kawai (N.), University of Kyoto, Department of Geology, Kyoto.

KIKUGH (K.), Wayne State University, Department of Physics, Detroit 2 (Michigan).

Kotani (M.), Department of Physics, Faculty of Science, Tokyo.

NagamiYa (T.), Faculty of Science, Osaka University, Nakanoshima, Osaka.

\section{Norvège}

Riste (T.), Joint Establishment for Nuclear Energy Research, P. O. 175, Lillestrom.

\section{Pays-Bas}

Bosman (A. J.), Natuurkundig Laboratorium der Universiteit van Amsterdam, Amsterdam.

Casimir (H. B. G.), Philips Research Laboratories, Eindhoven.

Enz (U.), Phllips Research Laboratories, Eindhoven.

Gersdorf (R.), Natuuirkundig Laboratorium, Amsterdam. 
Heснт (C. E.), Institut voor Theoretische Physica, Universiteit van Amsterdam, Amsterdam-C.

Kasteleijn (P. W.), Koninklijke Shell-Laboratorium, Postbus 3003, Amsterdam-N.

Kосн (J. J.), Philips Research Laboratories, Eindhoven.

Lotgering (F.), Philips Research Laboratories, Eindhoven.

Poulis (N. J.), Kamerlingh Onnes Laboratorium, Nieusteeg 18, Leiden.

Rathenau (G. W.), Natuurkundig Laboratorium, Plantage Muidergracht 6, Amsterdam.

Snieder (J.), Physich Laboratorium, Rijksverdedigings-organisatie T. N. O., La Haye.

Sterrett (K. F.), Kamerlingh Onnes Laboratorium, Nieuwsteeg 18, Leiden.

Van Reijen (L. L.), Koninklijke, Shell Laboratorium, Postbus 3003, Amsterdam.

Verschoor, Philips Research Laboratories, Eindhoven.

Verweel (J.), Philips Laboratories, Eindhoven.

de Vries (G.), Natuurkundig Laboratorium, Amsterdam.

Zylstra (H.), Rulstraat 22, Geldrop.

\section{Pologne}

Leibler (Caroline), Institut de Physique, Politechnika, Koszykowa 75, Varsovie.

Szczeniowski (S.), Polska Akademia Nauk, Instytut Fizyki, Fredy 10, Poznan.

\section{Suisse}

Ascher (E.), Battelle Memorial Institute, CarougeGenève.

BerGer (L.), Université de Lausanne, Lausanne.

Croisier (A.), I. B. M., Forschungslaboratorium, Zürichstrasse 108, Adliswill-Zürich.

Hulliger (F.), Physikal Institut der Eidg. Technische Hochschule Zürich, Zürich.

KinberG (C.), I. B. M., Forschungslaboratorium, Zürichstrasse 108, Adliswill-Zürich.

Methfessel (S.), I. B. M., Forschungslaboratorium, Zürichstrasse 108, Adliswil-Zürich.

Proebster (W.), I. B. M., Forschungslaboratorium, Zürichstrasse 108, Adliswil-Zürich.

Schmid (H.), Département de Métallurgie, Battelle, Memorial Institute, 7, Route de Drize, CarougeGenève.

Speiser (A. P.), I. B. M., Research Laboratory Zürich, Zürichstrasse 108, Adliswill-Zürich.

Staub (H. H.), Physikalisches Institut der Universität, Rämistrasse 69 , Zürich.

Szasz (G.), Scientific Representative Europe General Electric Company, Pelikanstrasse 37, Zürich 11.

\section{Tchécoslovaquie}

Kaczér (J.), Institut of Physics, Czechosl. Academy of Science, Vinicna ul. 7, Praha II.

Valenta (L.), Fakulta Technické a Jaderné Fysiky, University Karlovy, Praha.

\section{U. R. S. S.}

Kondorsky (E.), Université de Moscou, Laboratoire de Magnétisme, Moscou.

Smolensky (G.), Institut de Semiconducteurs, Leningrad.

Vonsovsky, Sverdlovsk.

\section{France}

Aleonard (R.), Laboratoire d'Électrostatique et de Physique du Métal, Grenoble.

Angeli, I. R. S. I. D., 185, rue Président-Roosevelt, Saint-Germain-en-Laye (Seine-et-Oise).

Asch (G.), Institut de Physique, Strasbourg.

Ayant (Y.), Université de Grenoble, Faculté des Sciences, Grenoble.

Barbier (J. C.), Université de Grenoble, Faculté des Sciences, Grenoble.

Bizette (H.), Institut de Physique, Nancy.

Blandin (A.), Centre de Recherches Métallurgiques de l'E. N. S. des Mines de Paris, 60, bd SaintMichel, Paris (VIe).

Bonnet (G.), Centre d'Études Nucléaires de Grenoble.

Brissonneau (P.), Université de Grenoble, Faculté des Sciences.

Burger (J. P.), Institut de Physique, Strasbourg.

Cohen (J.), Université de Grenoble, Faculté des Sciences.

Colombani, Faculté des Sciences, rue du Gaillon, Caen.

Conte (R.), Laboratoire d'Électrostatique et de Physique du Métal, Grenoble.

Danan (H.), Institut de Physique, Strasbourg.

Daniel (E.), Centre de Recherches Métallurgiques de l'E. N. S. des Mines de Paris, Paris (VIe).

Dautreppe (D.), Centre d'Études Nucléaires de Grenoble.

Delyon (G.), Société des Lignes Télégraphiques et Téléphoniques, Conflans-Sainte-Honorine (Seineet-Orse).

Deschamps (R.), Laboratoire d'Électrostatique et de Physique du Métal, Grenoble.

Dreyfus (B.), Université de Grenoble, Faculté des Sciences.

Epelboin, Laboratoire de Physique enseignement, La Sorbonne, Paris (Ve).

Erickson, Centre d'Études Nucléaires de Saclay, Gif-sur-Yvette (Seine-et-Oise).

Ferguson (E. T.), Laboratoire d'Électrostatique et de Physique du Métal, Grenoble. 
Forrer, Institut de Physique, Strasbourg.

Friedel (J.), Centre de Recherches Métallurgiques de l'E. N. S. des Mines de Paris, Paris (VIe).

Gariod, Centre d'Études Nucléaires de Grenoble.

DE Gennes, Centre d'Études Nucléaires de Saclay, Gif-sur-Yvette (Seine-et-Oise).

Gouraud, Université de Caen, Faculté des Sciences.

Guiot-Guillain (G.), E. N. S. de Chimie, 2, rue Goethe, Strasbourg.

Guillaud (Ch.), Laboratoires de Bellevue, 1, place Aristide-Briand, Bellevue (Seine-et-Oise).

Herpin (A.), Centre d'Études Nucléaires de Saclay. JACROT (B.), Centre d'Études Nucléaires de Saclay. Lensen, Université de Lille, Faculté des Sciences.

Lewy-Bertaut (E. F.), Laboratoire d'Électrostatique et de Physique du Métal, Grenoble.

LovichI, Société Kodak-Pathé, 30, rue de Vincennes.

Meriel, Centre d'Études Nucléaires de Saclay.

Meyer (A. J. P.), Institut de Physique, Strasbourg.

Moser, Centre d'Études Nucléaires de Saclay.

NÉEL (L.), Université de Grenoble, Faculté des Sciences.

NuguYEN-VAN-DANG, Laboratoire d'Électrostatique et de Physique du Métal, Grenoble.

Nicolas, 79, bd Haussmann, Paris (8e), T. S. F.

Paulevé (J.), Centre d'Études Nucléaires de Saclay.

Paulus, Laboratoire de Bellevue, Bellevue (Seineet-Oise).
Pauthenet (R.), Université de Grenoble, Faculté des Sciences.

Petis (J.), Institut de Chimie, 2, rue Grothe, Strasbourg.

Pierrot (A.), Société des Lignes Télégraphiques et Téléphoniques, Conflans-Sainte-Honorine (Seineet-Oise).

Pomey (G.), I. R. S. I. D., 185, rue PrésidentRoosevelt, Saint-Germain-en-Laye (S.-et-O.).

RoBieux, Compagnie Générale de Télégraphie sans Fil, Domaine de Corbeville, Orsay (S.-et-O.)

SANchez-Giron (V.), Laboratoire d'Électrostatique et de Physique du Métal, Grenoble.

Soutif (M.), Université de Grenoble, Faculté des Sciences.

Thellier, Institut de Physique du Globe, Observatoire Géophysique du Parc Saint-Maur, SaintMaur-des-Fossés (Seine).

Thourel, Compagnie Générale de Télégraphie sans Fil.

Vautier (R.), Ląboratoire de Bellevue, Bellevue (Seine-et-Oise).

Villers (G.), Laboratoire de Bellevue, Bellevue (Seine-et-Oise).

VERGNE (R.), Laboratoire d'Électrostatique et de Physique du Métal, Grenoble.

WeIL (L.), Université de Grenoble, Faculté des Sciences.

Wending (R.), Institut de Physique, Strasbourg.

YAMADA (O.), Laboratoire d'Électrostatique et de Physique du Métal, Grenoble. 